BULL. AUSTRAL. MATH. SOC.

VOL. 5 (1971), 357-362.

\title{
On a class of power-associative periodic rings
}

\author{
J.A. Loustau
}

\begin{abstract}
A power-associative ring $A$ is called a p-ring provided there exists a prime $p$ so that for every $x$ in $A, x^{p}=x$ and $p x=0$. It is shown that if $A$ is such a ring with $p \neq 2$, then $A$ is isomorphic to a subdirect sum of copies of $G F(p)$, the Galois field with $p$ elements.
\end{abstract}

\section{Introduction}

A power-associative ring $A$ is called a p-ring provided there exists a prime $p$ so that for every $x$ in $A, x^{p}=x$ and $p x=0$. It is well known that any associative $p$-ring is commutative and is isomorphic to a subdirect sum of copies of $G F(p)$ (see for example, [3, p. 144]). In this paper we will extend this result to power-associative $p$-rings with $p \neq 2$. Stated formally we have:

THEOREM. Let $A$ be a power-associative p-ring with $p \neq 2$, then $A$ is associative and commutative. Thus, $A$ is a subdirect sum of copies of $\mathrm{GF}(p)$.

Before proceeding we need the following terminology. Let $A$ be an algebra over a field $F$ not of characteristic 2 . Then $A^{+}$will denote the algebra which is the same set as $A$ with addition and scalar multiplication defined as in $A$ and multiplication defined by $x \cdot y=\frac{1}{2}(x y+y x)$, where juxtaposition denotes the product in $A$. Hence $A^{+}$ is a commutative algebra which is power-associative if $A$ is

Received 22 June 1971. 


\section{power-associative.}

The proof of the theorem will proceed as follows. We begin by letting $A$ be a power-associative $p$-ring of characteristic not 2 and show that $A^{+}$is a Jordan ring. The argument used here is similar to the one employed by Albert which showed that for any finite strictly power-associative division ring $D, D^{+}$is a Jordan algebra [5, p. 133]. Next we shall show that $A^{+}$is associative. For this purpose we first apply the Shirshov-Cohn Theorem and then the Vandermonde determinant argument employed by Forsythe and McCoy which showed that associative p-rings are commutative [3, p. 144]. The proof is completed by showing that if $A$ is a power-associative algebra of characteristic not 2 such that for every $a$ in $A$ there exists an integer $n(a)>1$, depending on $a$, with $a^{n(a)}=a$, then $A$ is commutative and associative provided $A^{+}$ is associative.

\section{Proof}

In order to show that $A^{+}$is a Jordan algebra, it is necessary to show that $\left(x, y, x^{2}\right)$, the associator in $x, y$, and $x^{2}$, is zero for every $x$ and $y$ in $A^{+}$. For this purpose we look at the ring $\langle x\rangle$ generated by $x$. Clearly we may suppose that $x \neq 0$. Since $A$ is power-associative, then $\langle x\rangle$ is associative. Also since $A$ is a p-ring, it follows that $\langle x\rangle$ is a finite, semi-simple algebra over GF(p). Thus, $\langle x\rangle$ is equal to the direct sum of a finite number of copies of GF(p).

Hence if $(x)=\sum_{i=1}^{n} F_{i}$, with $F_{i}=G F(p)$ for every $i=1, \ldots, n$, then there are elements $\lambda_{i}$ and $\mu_{j} \in G F(p), i, j=1, \ldots, n$, with $x=\sum_{i=1}^{n} \lambda_{i} e_{i}$ and $x^{2}=\sum_{j=1}^{n} \mu_{j} e_{j}$, where $e_{i}$ is the identity of $F_{i}$. Also it is clear that $\left\{e_{i}\right\}_{i=1}^{n}$ is a set of orthogonal idempotents in $A^{+}$. Now with this representation for $x$ and $x^{2}$ it follows that 


$$
\begin{aligned}
\left(x, y, x^{2}\right) & =\left(\sum_{i=1}^{n} \lambda_{i} e_{i}, y, \sum_{j=1}^{n} \mu_{j} e_{j}\right) \\
& =\sum_{i, j=1}^{n} \lambda_{i} \mu_{j}\left(e_{i}, y, e_{j}\right) \\
& =\sum_{\substack{i, j=1 \\
i \neq j}}^{n} \lambda_{i} \mu_{j}\left(e_{i}, y, e_{j}\right),
\end{aligned}
$$

since $A^{+}$is commutative and hence flexible. However, Albert has shown that in a commutative, power-associative algebra $R,\left(e, r, e^{\prime}\right)=0$ for every $r \in R$ and every pair of orthogonal idempotents $e, e^{\prime}[5$, Lemma 5.2 , p. 133]. Thus, indeed $\left(x, y, x^{2}\right)=0$, and $A^{+}$is Jordan.

Next we claim that the Jordan algebra $A^{+}$is associative. First of all note that because $A^{+}$has no nonzero nilpotent elements, it suffices to show that $A^{+}$is alternative. This is the case due to the well-known fact that any commutative, alternative ring without nonzero nilpotent elements is associative [4, Lemma 3, p. 1175]. Hence, we only need show that if $A^{+}$is generated by two elements, then it is associative. Therefore, we can suppose by the Shirshov-Cohn Theorem that $A^{+}$is a special Jordan algebra.

Let $\left(S\left(A^{+}\right), \sigma_{u}\right)$ be the special universal envelope of $A^{+}$. Then we have that $\sigma_{u}$ is an injection mapping and $A^{+}$can be assumed to be contained in $S\left(A^{+}\right)^{+}$. Now $S\left(A^{+}\right)$has the following properties. It is an associative algebra with identity generated by $\left\{x: x \in A^{+} \cup \mathrm{GF}(p)\right\}$. Also if $x, y \in A^{+}$with $x . y$ their product in $A^{+}$and $x y$ their product in $S\left(A^{+}\right)$, then $x \cdot y=\frac{1}{2}(x y+y x)$. Therefore, to show that $A^{+}$is associative it suffices to show that $S\left(A^{+}\right)$is commutative, since in this case $A^{+}$is a subalgebra of $S\left(A^{+}\right)^{+}=S\left(A^{+}\right)$. Clearly $S\left(A^{+}\right)$is cormutative if and only if $x y=y x$ for every $x, y \in A^{+}$. 
Now with $x$ and $y$ in $A^{+}$, we have in $S\left(A^{+}\right)$

$$
(x+y)^{p}=x^{p}+y^{p} \text {. }
$$

Therefore in $S\left(A^{+}\right)$,

$$
A_{1}+A_{2}+\ldots+A_{p-1}=0
$$

where $A_{i}$ is the sum of all words in the expansion of $(x+y)^{p}$ in which $y$ appears $i$ times and $x$ appears $(p-i)$ times. Then substituting $\lambda y$ for $y$ in ( 1 ) for any $\lambda \in G F(p)$ we have

$$
\lambda A_{1}+\lambda^{2} A_{2}+\ldots+\lambda^{p-1} A_{p-1}=0 \text {. }
$$

Thus, if $m$ denotes the determinant of the matrix

$$
\left[\begin{array}{cccc}
1 & 1 & \cdots & 1 \\
2 & 2^{2} & \cdots & 2^{p-1} \\
\vdots & \vdots & & \vdots \\
(p-1) & (p-1)^{2} & \ldots & (p-1)^{p-1}
\end{array}\right]
$$

and if $m_{1}, \ldots, m_{p-1}$ denote the co-factors of the elements of the first column of the above matrix, then by multiplying the $\lambda$-th equation in (2) by $m_{\lambda}$ and adding we have from an elementary property of determinants that $m A_{1}=0$. But since the above determinant is a Vandermonde determinant it follows that $m$ and $p$ are relatively prime. Hence $A_{1}=0$. Now by an easy calculation we have

$$
0=x A_{1}-A_{1} x=x^{p} y-y x^{p}=x y-y x .
$$

Therefore $S\left(A^{+}\right)$is commutative, and hence $A^{+}$is associative.

It remains to show that $A$ is commutative, since then $A=A^{+}$and the result will follow. For this purpose we have the following lemma.

LEMMA. Let $R$ be a power-associative algebra of characteristic not 2 such that for every $a$ in $R$ there exists a positive integer $n(a)>1$, depending on $a$, with $a^{n(a)}=a$. Then $R$ is commutative and 
associative if $R^{+}$is associative.

Proof. As above, it suffices to show that $R$ is commutative. Let $x$ and $y$ be in $R$ and look at the ring $B$ generated by $x$ and $y$. Since $B^{+}$is a finitely generated, associative, commutative ring, without nonzero nilpotent elements and satisfying the hypothesis of the lemma, then $B^{+}$is the direct sum of a finite number of Galois fields, that is,

$B^{+}=\sum_{i=1}^{n} F_{i}$ where each $F_{i}$ is a finite field. Hence to show that $A$ is commutative it suffices to show that $s t=t s$ for every $s \in F_{i}$ and $t \in F_{j}$ for some choice of $i$ and $j$. Also, we can suppose that neither $s$ nor $t$ is zero. If $i=j$, then there is a $z \in B$ and positive integers $\alpha$ and $\beta$ with $z^{\alpha}=s$ and $z^{\beta}=t$. So by the power-associativity of $B$, st $=t s$. If $i \neq j$, then we look at the following identity which holds in any power-associative algebra not of characteristic 2

$$
[a \cdot b, c]+[a \cdot c, b]+[b . c, a]=0
$$

for every $a, b, c \in B$ [5, p. 129]. (Here $a . b$ denotes the product in $B^{+}$and $[u, v]$ is the commutator in $B$ of $u$ and $\left.v.\right)$ since $B^{+}$is the direct sum of the fields $F_{i}, i=1, \ldots, n$, then $u . v=0$ for every $u \in F_{i}, v \in F_{j}, i \neq j$. Then by setting $a=s, b=s^{n(s)-1}, c=t$ in (3) it follows that the last two commutators in (3) are zero. Hence

$$
0=\left[s \cdot s^{n(s)-1}, t\right]=[s, t] .
$$

Therefore, $B$ is indeed commutative, and since $x$ and $y$ were chosen arbitrarily, $R$ is also commutative. This completes the proof of the lemma and also the proof of the theorem.

\section{References}

[1] Jerry Goldman and Seymour Kass, "Linearization in rings and algebras", Amer. Math. Monthly 76 (1969), 348-355. 
[2] Nathan Jacobson, Stmucture and representations of Jordan aZgebras (Colloquium Publ. 39. Amer. Math. Soc., Providence, Rhode Island, 1968).

[3] Neal H. McCoy, Rings and ideals (Carus Mathematical Monographs, No. 8. Mathematical Association of America; John Wiley \& Sons, New York, 1948).

[4] Kevin McCrimmon, "Finite power-associative division rings", Proc. Amer. Math. Soc. 17 (1966), 1173-1177.

[5] Richard D. Schafer, An introduction to nonassociative algebras (Academic Press, New York, London, 1966).

University of California at Santa Barbara, Santa Barbara, California, USA. 